\title{
ON THE SOLUTION OF INTEGRAL EQUATIONS WITH A GENERALIZED CAUCHY KERNEL*
}

BY

\author{
A. C. KAYA AND F. ERDOGAN \\ Lehigh University, Bethlehem
}

\begin{abstract}
In this paper a certain class of singular integral equations that may arise from the mixed boundary value problems in nonhomogeneous materials is considered. The distinguishing feature of these equations is that in addition to the Cauchy singularity, the kernels contain terms that are singular only at the end points. In the form of the singular integral equations adopted, the density function is a potential or a displacement and consequently the kernel has strong singularities of the form $(t-x)^{-2}, x^{n-2}(t+x)^{n}$ $(n \geqslant 2,0<x, t<b)$. The complex function theory is used to determine the fundamental function of the problem for the general case and a simple numerical technique is described to solve the integral equation. Two examples from the theory of elasticity are then considered to show the application of the technique.
\end{abstract}

1. Introduction. In elasticity or in potential theory if the medium contains a planar imperfection representing a discontinuity in displacements or potential (e.g., a crack, a plane insulation, a barrier), or in stress or flux vector (e.g., a plane inclusion, a distributed source), the related mixed boundary value problem may be formulated in terms of either a system of dual integral equations [1] or a singular integral equation [2,3]. If the "cut" corresponding to the plane of potential or flux discontinuity is fully embedded in a homogeneous component in the medium, it is known that the dominant part of the integral equation contains only a simple Cauchy kernel associated with the corresponding infinite domain and the remaining geometry of the medium is represented by a Fredholm kernel. On the other hand, if the medium is nonhomogeneous with discontinuous material parameters and if the cut intersects such a plane of discontinuity, then it is also known that the dominant kernel of the integral equation contains other singular terms in addition to the Cauchy kernel [3]. These kernels (which are sometimes known as the generalized Cauchy kernels) become unbounded as the variables approach the end of the cut responding to the point of intersection with the bimaterial interface.

\footnotetext{
* Received March 19, 1986. This work was supported by NSF under the (irant MEA-8414477 and by NASA-Langley under the Grant NGR 39-007-011.
} 
In the case of singular integral equations with ordinary Cauchy kernel the solution of the problem is quite straightforward and there are very highly efficient numerical techniques to obtain it. Most of these simple techniques are based on a variety of quadrature formulas developed for singular integrals (see, for example [4]). Another such technique introducing further computational economy as well as maintaining the basic numerical simplicity in the applications was discussed in a recent article [5]. The main features of this technique are (i) potential rather than flux type quantities are used as the unknown functions in deriving the integral equations resulting in a dominant kernel with a singularity stronger than that of the standard Cauchy kernel, (ii) the unknown function is expressed in terms of the fundamental solution of the problem and a bounded function containing a set of unknown coefficients, (iii) interpreting the integrals with strong singularities in the Hadamard sense, some useful formulas are developed to evaluate the integrals corresponding to the dominant part of the integral equation in closed form, and (iv) an appropriate collocation method is used to solve the resulting functional equation for the unknown coefficients. In [5] this method was used to solve also a special case of singular integral equations with a generalized Cauchy kernel, namely that of a cut intersecting a free boundary, and was shown to have distinct advantages over the conventional quadrature methods with regard to accuracy and computer time.

In this paper the mixed boundary value problems leading to singular integral equations with a generalized Cauchy kernel are reconsidered by formulating them in terms of "potentials" as the unknown functions. The terms in the resulting dominant kernel would then have strong singularities and must be interpreted in the Hadamard sense. It is shown that the complex function theory can again be used to determine the fundamental solution. The main objective of the paper is to develop a simple and efficient technique for solving singular integral equations with generalized Cauchy kernels.

2. Integral equations. Consider first the simple problem in potential theory described in Fig. 1. In the terminology of elasticity the problem is one of antiplane shear loading of a nonhomogeneous medium which consists of two bonded half spaces with shear moduli $\mu_{1}$ and $\mu_{2}$. The medium contains a crack along $\theta=\pi, a<r<b$ and, in the perturbation problem considered, the crack surface traction $\sigma_{1 \theta z}(r, \pi)=q(r)$ is the only nonzero external load. If $w_{1}$ and $w_{2}$ are the $z$-component of the displacement vector in materials 1 and 2 , respectively, it was shown that (see, for example, $[6,7])$ the differential equations and boundary conditions

$$
\begin{gathered}
\nabla^{2} w_{1}=0, \quad \nabla^{2} w_{2}=0, \\
w_{1}=w_{2}, \quad \sigma_{1 \theta z}=\sigma_{2 \theta z} \quad\left(\theta=\frac{\pi}{2}, \quad 0 \leqslant r<\infty\right), \\
w_{2}(r, 0)=0 \quad(0 \leqslant r<\infty), \\
w_{1}(r, \pi)=0 \quad(0 \leqslant r<a, \quad b<r<\infty), \\
\sigma_{1 \theta z}(r, \pi)=q(r) \quad(a<r<b),
\end{gathered}
$$




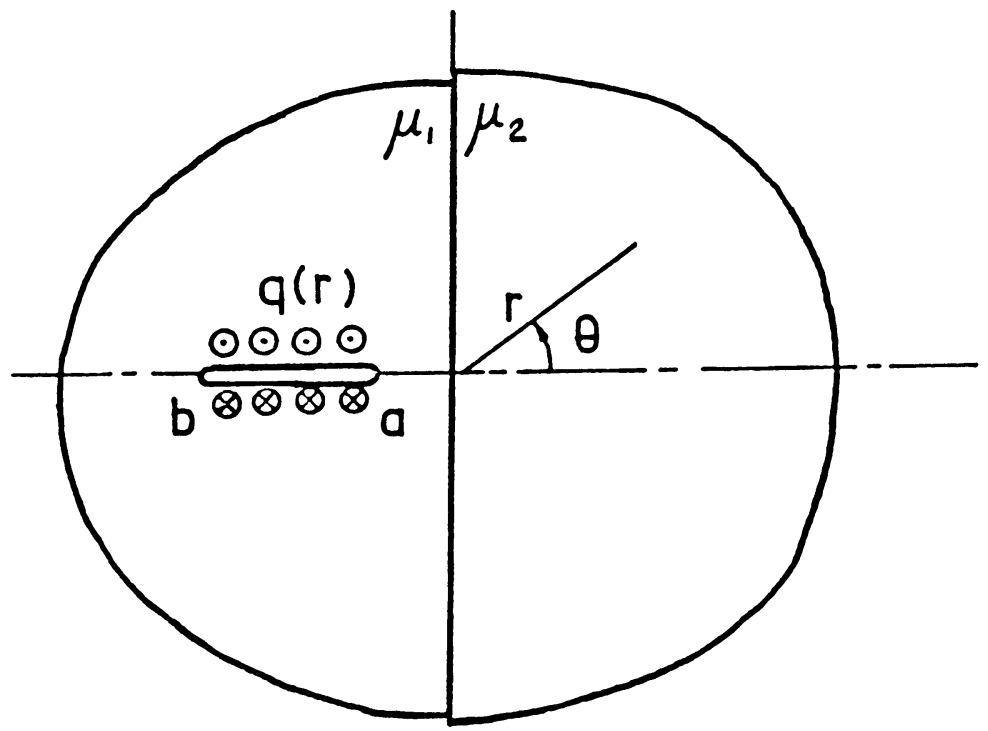

FIG. 1 Bonded Half-Planes (Antiplane Shear Loading)

would give the following integral equation:

$$
\frac{1}{\pi} f_{a}^{b}\left(\frac{1}{t-r}+\frac{\lambda}{t+r}\right) \psi(t) d t=\frac{2}{\mu_{1}} q(r) \quad(a<r<b),
$$

where

$$
\begin{gathered}
\psi(r)=\frac{\partial}{\partial r}\left[w_{1}(r, \pi+0)-w_{1}(r, \pi-0)\right], \\
\lambda=\left(\mu_{1}-\mu_{2}\right) /\left(\mu_{1}+\mu_{2}\right)
\end{gathered}
$$

and, because of symmetry, only one half of the medium is considered. We note that for $a=0$ and $\mu_{1} \neq \mu_{2}$ the term $\lambda(t+r)^{-1}$ becomes unbounded for $r=0=t$ and the kernel is one of generalized Cauchy type.

Integrating (5) by parts and using (4a) it may be seen that

$$
\frac{1}{\pi} f_{a}^{b}\left[\frac{1}{(t-r)^{2}}+\frac{\lambda}{(t+r)^{2}}\right] w(t) d t=\frac{2}{\mu_{1}} q(r) \quad(a<r<b),
$$

where

$$
w(t)=w_{1}(t, \pi+0)-w_{1}(t, \pi-0) .
$$

The integrals in (5) and (8) are to be interpreted in Cauchy principal value and Hadamard sense, respectively [5]. The integral equation can also be obtained by using $w(t)$ rather than $\psi(t)$ as the unknown function and, for example, following the procedure outlined in [6]. It may be noted that in terms of $w$ some of the stress components of physical interest may be expressed as

$$
\sigma_{2 \theta z}(r, 0)=-\frac{\mu_{1} \mu_{2}}{\mu_{1}+\mu_{2}} \frac{1}{\pi} \int_{a}^{b} \frac{w(t)}{(t+r)^{2}} d t \quad(0<r<\infty),
$$




$$
\begin{gathered}
\sigma_{1 \theta z}(r, \pi / 2)=\sigma_{2 \theta z}(r, \pi / 2)=-\frac{\mu_{1} \mu_{2}}{\mu_{1}+\mu_{2}} \frac{1}{\pi} \int_{a}^{b} \frac{r t}{\left(t^{2}+r^{2}\right)^{2}} w(t) d t \quad(0<r<\infty), \\
\sigma_{1 \theta z}(r, \pi)=\frac{\mu_{1}}{2 \pi} \int_{a}^{b}\left[\frac{1}{(t-r)^{2}}+\frac{\lambda}{(t-r)^{2}}\right] w(t) d t \quad(0 \leqslant r<a, b<r<\infty) .
\end{gathered}
$$

Similarly, for the crack geometry shown in Fig. 1, under the following symmetric in-plane loading condition:

$$
\sigma_{1 \theta \theta}(r, \pi)=-p(r), \quad \sigma_{1 r \theta}(r, \pi)=0 \quad(a<r<b),
$$

the integral equation may be obtained as (see, for example, [8] for the procedure)

$$
f_{a}^{b} \frac{v(t)}{(t-r)^{2}} d t+\int_{a}^{b} K(r, t) v(t) d t=-\pi \frac{1+\kappa_{1}}{2 \mu_{1}} p(r) \quad(a<r<b),
$$

where

$$
\begin{gathered}
v(t)=u_{1 \theta}(t, \pi+0)-u_{1 \theta}(t, \pi-0) \quad(a<t<b), \\
K(r, t)=\frac{c_{1}}{(t+r)^{2}}+\frac{2 c_{2} r}{(t+r)^{3}}+\frac{3 c_{3} r^{2}}{(t+r)^{4}}, \\
c_{1}=\frac{1}{2}\left[1-\frac{\left(1+\kappa_{1}\right) m}{m+\kappa_{2}}-\frac{3(1-m)}{1+m \kappa_{1}}\right], \\
c_{2}=\frac{6(1-m)}{1+m \kappa_{1}}, \quad c_{3}=-\frac{4(1-m)}{1+m \kappa_{1}}, \quad m=\mu_{2} / \mu_{1} .
\end{gathered}
$$

In the above formulation $\mu_{1}$ and $\mu_{2}$ are the shear moduli, $\kappa_{i}=3-4 \nu_{i}$ for plane strain, $\kappa_{i}=\left(3-\nu_{i}\right) /\left(1+\nu_{i}\right)$ for plane stress $(i=1,2)$, and $\nu_{1}$ and $\nu_{2}$ are the Poisson's ratio of the two materials. Once the integral equation is solved, in this problem too all the desired field quantities may be expressed in terms of $v(t)$ and the corresponding kernels. For example, the cleavage stresses along the planes $\theta=0, \theta=\pi / 2$, and $\theta=\pi$ may be written as [8]

$$
\begin{gathered}
\sigma_{2 \theta \theta}(r, 0)=\frac{\mu_{2}}{\pi} \int_{a}^{b}\left[\left(\frac{3}{m+\kappa_{2}}-\frac{1}{1+m \kappa_{1}}\right) \frac{1}{(t+r)^{2}}\right. \\
\left.\quad+\left(\frac{1}{1+m \kappa_{1}}-\frac{1}{m+\kappa_{2}}\right) \frac{4 r}{(t+r)^{3}}\right] v(t) d t \quad(0<r<\infty), \\
\sigma_{1 \theta \theta}(r, \pi / 2)=\sigma_{2}(r, \pi / 2)=\frac{\mu_{2}}{\pi} \int_{a}^{b}\left\{\frac{1}{m+\kappa_{2}}\left[\frac{1}{t^{2}+r^{2}}-\frac{2 r^{2}}{\left(t^{2}+r^{2}\right)^{2}}\right]\right. \\
\left.+\frac{1}{1+m \kappa_{1}}\left[\frac{1}{t^{2}+r^{2}}-\frac{14 r^{2}}{\left(t^{2}+r^{2}\right)^{2}}+\frac{16 r^{4}}{\left(t^{2}+r^{2}\right)^{3}}\right]\right\} v(t) d t \quad(0<r<\infty),
\end{gathered}
$$




$$
\sigma_{1 \theta \theta}(r, \pi)=\frac{2 \mu_{1}}{\pi\left(1+\kappa_{1}\right)} \int_{a}^{b}\left[\frac{1}{(t-r)^{2}}+K(r, t)\right] v(t) d t \quad(0<r<a, b<r<\infty),
$$

where $K(r, t)$ is given by (16).

In the problems given by the integral equations (8) and (14) for a crack embedded in a homogeneous phase, (i.e., for $a>0$ ), the dominant kernel consists of $(t-r)^{-2}$, the fundamental function of the integral equations is $(b-r)^{1 / 2}(r-a)^{1 / 2}$, and the solutions may be obtained by using the technique described in [5]. On the other hand, if $a=0$ clearly the kernels are of the generalized Cauchy type and, before attempting to solve the problem, the fundamental function of the integral equation needs to be determined. This may easily be done by using certain properties of Cauchy integrals [2].

3. The fundamental function. For $a=0$ we note that the general form of the integral equations (8) and (14) is

$$
\frac{1}{\pi} f_{0}^{b}\left[\frac{1}{(t-x)^{2}}+\sum_{k=2}^{K} \frac{B_{k-2} x^{k-2}}{(t+x)^{k}}\right] f(t) d t=p(x) \quad(0<x<b) .
$$

To determine the behavior of $f(t)$ near and at the end points, following [2] we define $f(t)=g(t) t^{\beta}(b-t)^{\alpha}=g(t) e^{-\pi i \alpha} t^{\beta}(t-b)^{\alpha}$

$$
(\operatorname{Re}(\alpha, \beta)>0, g(0) \neq 0, g(b) \neq 0,0<t<b),
$$

where $g(t)$ is bounded in the closed interval $[0, b]$ and the function $t^{\beta_{1}}(b-t)^{\alpha_{1}}$ constituting the leading term is generally known as the fundamental function of the integral equation $\left(\alpha=\alpha_{1}, \alpha_{2}, \ldots, \quad \beta=\beta_{1}, \beta_{2}, \ldots, 0<\operatorname{Re}\left(\alpha_{1}\right)<\operatorname{Re}\left(\alpha_{2}\right), \ldots, 0<\operatorname{Re}\left(\beta_{1}\right)<\right.$ $\left.\operatorname{Re}\left(\beta_{2}\right), \ldots\right)$.

Defining the sectionally holomorphic function

$$
F(z)=\frac{1}{\pi} \int_{0}^{b} \frac{f(t)}{t-z} d t \quad(z=x+i y),
$$

it may be seen that

$$
\frac{1}{\pi} \int_{0}^{b} \frac{f(t)}{(t-z)^{2}} d t=G(z)=\frac{d}{d z} F(z)=\frac{e^{-\pi i \alpha}}{\pi} \int_{0}^{b} t^{\beta}(t-b)^{\alpha} \frac{g(t)}{(t-z)^{2}} d t .
$$

From (24) the asymptotic behavior of $G(z)$ near the ends may be expressed as

$$
G(z)=-g(0) b^{\alpha} \frac{\beta e^{-\pi i \beta}}{\sin \pi \beta} z^{\beta-1}+g(b) b^{\beta} \frac{\alpha}{\sin \pi \alpha}(z-b)^{\alpha-1}+G_{0}(z),
$$

where $G_{0}(z)$ is bounded everywhere except possibly the end points near which it may have the following behavior:

$$
\left|G_{0}(z)\right|<C_{i}\left|z-d_{i}\right|^{p_{1}-1} \quad\left(d_{1}=0, p_{1}>\operatorname{Re}(\beta) ; d_{2}=b, p_{2}>\operatorname{Re}(\alpha)\right) .
$$

First we note that if $z$ is not on the cut, then $G(z)$ is holomorphic and at a point $z=z_{0}=x e^{i \theta},(0<x<b)$, we can write

$$
\frac{1}{\pi} \int_{0}^{b} \frac{f(t)}{\left(t-z_{0}\right)^{2}} d t=G\left(z_{0}\right)=-g(0) b^{\alpha} \frac{\beta e^{i((\beta-1) \theta-\pi \beta]}}{\sin \pi \beta} x^{\beta-1}+G_{1}\left(z_{0}\right),
$$


where $G_{1}$ is bounded everywhere and near $z_{0}=0$ has a behavior similar to that of $G_{0}$. For example, if $\theta=\pi$ (i.e., if $z_{0}=-x$ ), by differentiating from (27) it may easily be shown that near the end $x=0$ we have

$$
\begin{array}{r}
\frac{1}{\pi} \int_{0}^{b} \frac{f(t)}{(t+x)^{k}} d t=(-1)^{k} \frac{\beta(\beta-1) \cdots(\beta-k+2)}{(k-1) ! \sin \pi \beta} g(0) b^{\alpha} x^{\beta+1-k}+O\left(x^{p_{1}+1-k}\right) \\
\quad\left(k \geqslant 2, x>0, p_{1}>\operatorname{Re}(\beta)\right) .
\end{array}
$$

Similarly, substituting $\theta=\pi / 2$ and $\theta=-\pi / 2$ (i.e., for $z_{0}=\mp i x$ ) and using

$$
\frac{1}{t^{2}+x^{2}}=\frac{1}{2 i x}\left(\frac{1}{t-i x}-\frac{1}{t+i x}\right), \quad \frac{t}{t^{2}+x^{2}}=\frac{1}{2}\left(\frac{1}{t-i x}+\frac{1}{t+i x}\right)
$$

it can be shown that

$$
\begin{aligned}
& \frac{1}{\pi} \int_{0}^{b} \frac{f(t)}{t^{2}+x^{2}} d t=\frac{1}{2 \cos \frac{\pi \beta}{2}} g(0) b^{\alpha} x^{\beta-1}+O\left(x^{p_{1}-1}\right) \quad\left(x>0, p_{1}>\operatorname{Re}(\beta)\right), \\
& \frac{1}{\pi} \int_{0}^{b} \frac{f(t)}{\left(t^{2}+x^{2}\right)^{k}} d t=(-1)^{k-1} \frac{(\beta-1)(\beta-3) \cdots(\beta+3-2 k)}{(k-1) ! 4 \cos \frac{\pi \beta}{2}} g(0) b^{\alpha} x^{\beta+1-2 k} \\
& +O\left(x^{p_{1}+1-2 k}\right) \quad\left(k \geqslant 2, x>0, p_{2}>\operatorname{Re}(\beta)\right) . \\
& \frac{1}{\pi} \int_{0}^{b} \frac{t f(t)}{\left(t^{2}+x^{2}\right)^{k}} d t=(-1)^{k} \frac{\beta(\beta-2) \cdots(\beta+4-2 k)}{(k-1) ! 4 \sin \frac{\pi \beta}{2}} g(0) b^{\alpha} x^{\beta+2-2 k} \\
& +O\left(x^{p_{1}+2-2 k}\right) \quad\left(k \geqslant 2, x>0, p_{2}>\operatorname{Re}(\beta)\right) .
\end{aligned}
$$

Next, by using the Plemelj formula [2]

$$
\frac{1}{\pi} f_{0}^{b} \frac{f(t)}{t-x} d t=\frac{1}{2}\left[F^{+}(x)+F^{-}(x)\right] \quad(0<x<b),
$$

we find

$$
\frac{1}{\pi} f_{0}^{b} \frac{f(t)}{(t-x)^{2}} d t=\frac{1}{\pi} \frac{d}{d x} f_{0}^{b} \frac{f(t)}{t-x} d t=\frac{1}{2}\left[G^{+}(x)+G^{-}(x)\right] \quad(0<x<b) .
$$

Thus, for example, using properties of the form

$$
\begin{aligned}
& {\left[Z^{\beta-1}\right]^{+}=\lim _{\theta \rightarrow+0}\left[x e^{i \theta}\right]^{\beta-1}=x^{\beta-1},} \\
& {\left[z^{\beta-1}\right]^{-}=\lim _{\theta \rightarrow 2 \pi-0}\left[x e^{i \theta}\right]^{\beta-1}=x^{\beta-1} e^{i 2 \pi \beta},}
\end{aligned}
$$

from (34) and (25) it follows that

$$
\begin{aligned}
\frac{1}{\pi} f_{0}^{\prime \prime} \frac{f(t)}{(t-x)^{2}} d t=-g(0) b^{\alpha} \beta \cot (\pi \beta) x^{\beta-1}-g(b) b^{\beta} \alpha \cot (\pi \alpha)(b-x)^{\alpha-1} \\
+O\left(x^{p_{1}-1},(b-x)^{p_{2}-1}\right), \quad\left(0<x<b, p_{1}>\operatorname{Re}(\beta), p_{2}>\operatorname{Re}(\alpha)\right) .
\end{aligned}
$$


Substituting now from (36) and (28) into the integral equation (21) we obtain

$$
\begin{aligned}
& -g(0) b^{\alpha} x^{\beta-1}\left\{\beta \cot (\pi \beta)-\sum_{k=2}^{K} B_{k-2}(-1)^{k} \frac{\beta(\beta-1) \cdots(\beta-k+2)}{(k-1) ! \sin \pi \beta}\right\} \\
& -g(b) b^{\alpha} \alpha \cot (\pi \alpha)(b-x)^{\alpha-1}+O\left(x^{p_{1}-1},(b-x)^{p_{2}-1}\right)=p(x) \quad(0<x<b) .
\end{aligned}
$$

Now, by observing that $g(0) \neq 0, g(b) \neq 0, p_{1}>\operatorname{Re}(\beta)>0, p_{2}>\operatorname{Re}(\alpha)>0$ and $p(x)$ is bounded in $0 \leqslant x \leqslant b$, from (37) multiplying both sides first by $x^{1-\beta}$ and letting $x \rightarrow 0$ then by $(b-x)^{1-\alpha}$ and letting $x \rightarrow b$, for the leading terms $0<\operatorname{Re}(\alpha, \beta)<1$ we obtain the following characteristic equations:

$$
\beta \cot \pi \beta-\sum_{k=2}^{K} B_{k-2}(-1)^{k} \frac{\beta(\beta-1) \cdots(\beta-k+2)}{(k-1) ! \sin \pi \beta}=0, \quad \cot \pi \alpha=0 .
$$

Note that for the end $x=b$ that is embedded in a homogeneous component of the medium, (38b) gives the known result $\alpha=1 / 2$.

For the integral equation (8), if $a=0$, by defining

$$
w(t)=g(t) t^{\beta}(b-t)^{\alpha} \quad(0<t<b, \operatorname{Re}(\alpha, \beta)>0),
$$

from (36) and (37) we find

$$
\cos \pi \beta-\lambda=0, \quad \cot \pi \alpha=0 .
$$

Similarly, for $a=0$ in the in-plane elasticity problem given by (14), if we let

$$
v(t)=g(t) t^{\beta}(b-t)^{\alpha} \quad(0<t<b, \operatorname{Re}(\alpha, \beta)>0)
$$

from (14), (16), (21), and (38a, b), it may be shown that

$$
-\cos \pi \beta+c_{1}+c_{2}(1-\beta)+c_{3}(1-\beta)(2-\beta) / 2=0, \quad \cot \pi \alpha=0 . \quad(42 \mathrm{a}, \mathrm{b})
$$

We also note that after determining the unknown function $g(t)$, the asymptotic behavior of the stress components around the end points $x=0$ and $x=b$ may be obtained by substituting from the asymptotic relations such as (28)-(30) and (36) into the expressions of stresses (e.g., (9)-(11) and (18)-(20)).

4. The numerical technique. To solve the integral equation (21) having the generalized Cauchy kernel we express the unknown function as

$$
f(t)=g(t) t^{\beta_{1}}(b-t)^{1 / 2}
$$

where $\beta_{1}$ is the smallest positive root of (38a) and $g(t)$ is an unknown bounded function. By defining the following normalized quantities

$$
t=\frac{b}{2}(1+\tau), \quad x=\frac{b}{2}(1+\rho), \quad f(t)=\frac{b}{2} \phi(\tau), \quad p(x)=p_{1}(\rho),
$$

from (21) we obtain

$$
\begin{gathered}
\frac{1}{\pi} f_{-1}^{1}\left[\frac{1}{(\tau-p)^{2}}+\sum_{k=2}^{K} \frac{B_{k-2}(1+\rho)^{k-2}}{(\tau+\rho+2)^{k}}\right] \phi(\tau) d \tau=p_{1}(\rho) \quad(-1<\rho<1), \\
\phi(\tau)=h(\tau)(1-\tau)^{1 / 2}(1+\tau)^{\beta_{1}} \quad(-1<\tau<1),
\end{gathered}
$$


where $h(\tau)$ is the new unknown function. As in [5], the numerical solution of (45) may now be obtained by expanding $h(\tau)$ into a series with known coordinate functions and unknown coefficients and by reducing the resulting functional equation to a system of algebraic equations through a suitable collocation technique. No particular system of coordinate functions seems to have a special advantage and a simple power series such as

$$
h(\tau) \cong \sum_{n=0}^{N} a_{n} \tau^{n} \quad(-1<\tau<1)
$$

seems to be quite adequate. Also, expressing the finite-part integral as [5]

$$
\begin{aligned}
f_{-1}^{1} \frac{\phi(\tau)}{(\tau-\rho)^{2}} d \tau=\int_{-1}^{1}\left[\phi(\tau)-\phi(\rho)-(\tau-\rho) \phi^{\prime}(\rho)\right] \frac{d \tau}{(\tau-\rho)^{2}} \\
-\phi(\rho)\left(\frac{1}{1-\rho}+\frac{1}{1+\rho}\right)+\phi^{\prime}(\rho) \log \left(\frac{1-\rho}{1+\rho}\right)
\end{aligned}
$$

seems to give very satisfactory results. ${ }^{1}$ Higher accuracy is obtained in the numerical solution if the density of collocation points is increased near the ends by, for example, selecting $\rho_{j}(j=0,1, \ldots, N)$ as the roots of Chebyshev polynomials.

Numerically the technique described above would give fast converging results provided

$$
\alpha_{i}-\alpha_{1} \geqslant 1, \quad \beta_{j}-\beta_{1} \geqslant 1 \quad(i, j=2,3, \ldots) .
$$

On the other hand, if the characteristic equations contain roots for which $\alpha_{i}-\alpha_{1}<1$ or $\beta_{j}-\beta_{1}<1(i, j=2,3, \ldots)$, then the numerical technique would converge slowly and would have to be modified. To see this we note that for the problem under consideration formulated in terms of a potential-type quantity $f(t)$ (see (21)), the physically acceptable roots of the characteristic equations are $\alpha_{1}, \alpha_{2}, \ldots$ and $\beta_{1}, \beta_{2}, \ldots$ with $\operatorname{Re}\left(\alpha_{i}, \beta_{j}\right)>0$ $(i, j=1,2, \ldots)$ and the solution may generally be expanded as

$$
f(t)=\sum_{i=1}^{\infty} \sum_{j=1}^{\infty} A_{i j}(b-t)^{\alpha_{i}} t^{\beta_{l}} \quad(0<t<b) .
$$

If we now identify the fundamental function as $(b-t)^{\alpha_{1}} t^{\beta_{1}}$ and express

$$
f(t)=g(t)(b-t)^{\alpha_{1}} t^{\beta_{1}},
$$

it is seen that

$$
g(t)=\sum_{i=1}^{\infty} \sum_{j=1}^{\infty} A_{i j}(b-t)^{\alpha_{t}-\alpha_{1}} t^{\beta_{1}-\beta_{1}}
$$

Since $\alpha_{i}>\alpha_{1}$ and $\beta_{j}>\beta_{1}(i, j=2,3, \ldots), g(t)$ would be bounded at the ends $t=0$ and $t=b$. On the other hand, if we consider the derivative of $g(t)$,

$$
g^{\prime}(t)=\sum_{i=1}^{\infty} \sum_{j=1}^{\infty} A_{i j}\left[\left(\alpha_{i}-\alpha_{1}\right)(b-t)^{\alpha_{1}-\alpha_{1}-1} t^{\beta_{1}-\beta_{1}}+\left(\beta_{j}-\beta_{1}\right)(b-t)^{\alpha_{1}-\alpha_{1}} t^{\beta_{1}-\beta_{1}-1}\right],
$$

\footnotetext{
'Note that for $\tau \rightarrow \rho$ the integrand on the right-hand side of $(48)$ is $\phi^{\prime \prime}(\rho) / 2$.
} 
it is seen that if there are characteristic roots for which

$$
\alpha_{i}-\alpha_{1}-1<0, \quad \beta_{j}-\beta_{1}-1<0 \quad(i, j=2,3, \ldots),
$$

then at the corresponding end point $g^{\prime}(t)$ would become unbounded and consequently $g(t)$ would be ill-defined. Since $g(0)$ and $g(b)$ invariably represent the magnitude of parameters of primary physical importance, it would then be necessary to modify the numerical procedure in order to compute these quantities more accurately.

As will be shown by the examples given in the next section, the convergence of the calculations can be improved quite considerably by introducing the following simple modification. Let (54) be valid for $\alpha_{i}(i=2,3, \ldots, L)$ and $\beta_{j}(j=2,3, \ldots, M)$. The corresponding terms are then embedded into the definition of $f(x)$ as

$$
f(t)=g_{11}(t)(b-t)^{\alpha_{1}} t^{\beta_{1}}+\sum_{i=2}^{L} g_{i 1}(t)(b-t)^{\alpha_{i}} t^{\beta_{1}}+\sum_{j=2}^{M} g_{1 j}(t)(b-t)^{\alpha_{1}} t^{\beta_{j}} \text {. }
$$

The functions $g_{i j}(t)$ are determined by again expanding them, for example, into power series with unknown coefficients and by using a suitable collocation method.

5. Examples. First we consider the mixed boundary value problem in potential theory for the nonhomogeneous medium described in Fig. 1 and formulated by the integral equation (8). For $a>0$, the fundamental function of the integral equation is $(b-t)^{1 / 2}(t-a)^{1 / 2}$, the unknown function can be expressed as

$$
w(t)=g(t)(b-t)^{1 / 2}(t-a)^{1 / 2} \quad(a<t<b),
$$

and the stress intensity factors or the parameters giving the strength of the flux singularity at the end points $r=a$ and $r=b$ may be defined and evaluated from

$$
\begin{aligned}
k_{3}(a) & =\lim _{r \rightarrow a} \sqrt{2(a-r)} \sigma_{1 \theta_{z}}(r, \pi) \quad(r<a) \\
& =\frac{\mu_{1}}{2} \lim _{t \rightarrow a} \frac{w(t)}{\sqrt{2(t-a)}}=\frac{\mu_{1}}{2} g(a) \sqrt{(b-a) / 2}, \\
k_{3}(b) & =\lim _{r \rightarrow b} \sqrt{2(r-b)} \sigma_{1 \theta z}(r, \pi) \quad(r>b) \\
& =\frac{\mu_{1}}{2} \lim _{t \rightarrow b} \frac{w(t)}{\sqrt{2(b-t)}}=\frac{\mu_{1}}{2} g(b) \sqrt{(b-a) / 2} .
\end{aligned}
$$

The problem is solved by introducing the normalized quantities

$$
\begin{array}{r}
t=\frac{b-a}{2} \tau+\frac{b+a}{2}, \quad r=\frac{b-a}{2} \rho+\frac{b+a}{2}, \quad w(t)=\frac{b-a}{2} \phi(\tau), \\
\phi(\tau)=h(\tau) \sqrt{1-\tau^{2}},
\end{array}
$$

and by letting

$$
h(\tau) \cong \sum_{n=0}^{N} a_{n} \tau^{n}, \quad \rho_{j}=\cos \left(\frac{2 j+1}{N+1} \frac{\pi}{2}\right) \quad(j=0,1, \ldots, N)
$$


The integrals that arise in this solution are evaluated by using the following formulas [5]:

$$
\begin{gathered}
f_{-1}^{1} \frac{\tau^{n} \sqrt{1-\tau^{2}}}{(\tau-\rho)^{2}} d \tau=\sum_{k=0}^{n} c_{k} \rho^{k} \quad(n \geqslant 0), \\
c_{k}=\left\{\begin{array}{l}
0, \text { for } n-k=\text { odd } \\
\frac{k+1}{2 \sqrt{\pi}} \frac{\Gamma\left(\frac{n-k-1}{2}\right)}{\Gamma\left(\frac{n-k+2}{2}\right)}, \text { for } n-k=\text { even. }
\end{array}\right.
\end{gathered}
$$

For a material pair corresponding to aluminum and epoxy and for a uniform shear stress $q(r)=q_{0}$ applied to the crack surface some results are given in Table 1, where the previous results, calculated by using a Gauss-Chebyshev quadrature technique [6], are also displayed. From the table it may be observed that the method seems to give rather good results even with a relatively small number of terms used for approximating the unknown function $h$, the convergence improves as the crack moves away from the interface, and at the crack tip near the interface the convergence for $\mu_{2}>\mu_{1}$ appears to be slower than the case for which $\mu_{1}>\mu_{2}$. Analytically, the difference in these two cases is only in the sign of $\lambda$ in (8) and, for $a>0$, is difficult to relate it to the convergence. However, as mentioned in the previous section, for $a=0$ there is a good reason for slow convergence and physically the two processes are clearly related.

Consider now the case of $a=0$ for which the characteristic equations are given by (40). For various material combinations the first two roots of (40a) are shown in Table 2. In this problem

$$
w(t)=g(t)(b-t)^{1 / 2} t^{\beta_{1}}
$$

and the stress intensity factors are defined by

$$
\begin{aligned}
& k_{3}(b)=\lim _{r \rightarrow b} \sqrt{2(r-b)} \sigma_{1 \theta z}(r, \pi), \\
& k_{3}(0)=\lim _{r \rightarrow 0} \sqrt{2} r^{1-\beta_{1}} \sigma_{2 \theta z}(r, 0) .
\end{aligned}
$$

TABLE 1. Stress intensity factors for a crack near an interface (anti-plane shear).

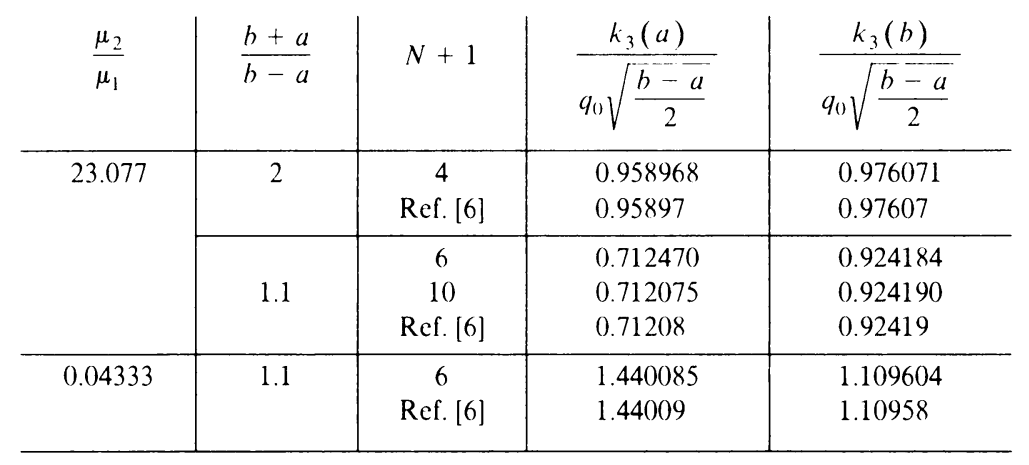


TABLE 2. The roots of the characteristic equation $\cos \pi \beta-\lambda=0$.

\begin{tabular}{lccc}
$\mu_{2} / \mu_{1}$ & $\lambda$ & $\beta_{1}$ & $\beta_{2}=2-\beta_{1}$ \\
\hline 0.04333 & 0.91694 & 0.13065 & 1.86935 \\
0.1 & 0.81818 & 0.19498 & 1.80502 \\
0.25 & 0.60000 & 0.29517 & 1.70483 \\
0.5 & 0.33333 & 0.39183 & 1.60817 \\
1. & 0.00000 & 0.50000 & 1.50000 \\
2. & -0.33333 & 0.60817 & 1.39183 \\
4. & -0.60000 & 0.70483 & 1.29517 \\
10. & -0.81818 & 0.80502 & 1.19498 \\
23.077 & -0.91694 & 0.86935 & 1.13065 \\
40. & -0.95122 & 0.90017 & 1.09983
\end{tabular}

Substituting now from (12) and (36) into (63) and from (10) and (28) into (64) and observing that $\sin \pi \beta_{1}=2 \sqrt{\mu_{1} \mu_{2}} /\left(\mu_{1}+\mu_{2}\right)$ we find

$$
\begin{aligned}
& k_{3}(b)=\frac{\mu_{1}}{2} \frac{b^{\beta_{1}}}{\sqrt{2}} g(b), \\
& k_{3}(0)=-\sqrt{\mu_{1} \mu_{2}} \beta_{1} g(0) \sqrt{b / 2} .
\end{aligned}
$$

For $q(r)=q_{0}$ a closed form solution of the integral equation (5) is given in [7] and [9] which, in terms of the normalized quantities

$$
\xi=t / b, \quad \psi_{1}(\xi)=\psi(t) \quad(0<t<b, \quad 0<\xi<1),
$$

may be expressed as [10]

$$
\begin{aligned}
\psi_{1}(\xi)=\frac{q_{0}}{\mu_{1} \sin \frac{\pi \gamma}{2}}\left[\left(\frac{\xi}{1+\sqrt{1-\xi^{2}}}\right)^{\gamma}\left(\frac{\gamma}{\sqrt{1-\xi^{2}}}+1\right)\right. \\
\left.+\left(\frac{\xi}{1+\sqrt{1-\xi^{2}}}\right)^{-\gamma}\left(\frac{\gamma}{\sqrt{1-\xi^{2}}}-1\right)\right], \quad \gamma=1-\beta_{1} .
\end{aligned}
$$

By observing that $\psi(t)=d w / d t$, from (68) the exact expressions of the stress intensity factors may be obtained as

$$
\begin{aligned}
& k_{3 e}(b)=-\frac{\mu_{1}}{2} \lim _{t \rightarrow b} \sqrt{2(b-t)} \psi(t)=-q_{0} \sqrt{b} \frac{1-\beta_{1}}{\cos \left(\pi \beta_{1} / 2\right)}, \\
& k_{3 e}(0)=-\sqrt{\mu_{1} \mu_{2} / 2} \lim _{t \rightarrow 0} t^{1-\beta_{1}} \psi(t)=\frac{q_{0}}{2^{\beta_{1}}} \sqrt{2 \mu_{2} / \mu_{1}} \frac{\beta_{1} b^{1-\beta_{1}}}{\cos \left(\pi \beta_{1} / 2\right)} .
\end{aligned}
$$

The numerical results given in Tables $3-5$ have been normalized as follows:

$$
\bar{k}_{3}(0)=\frac{k_{3}(0)}{q_{0}(b / 2)^{1-\beta_{1}}}, \quad \bar{k}_{3}(b)=\frac{k_{3}(b)}{q_{0} \sqrt{b / 2}} .
$$

Tables 3 and 4 show the calculated results obtained by assuming the solution of (8) in the form

$$
w(t)=g(t)(b-t)^{1 / 2} t^{\beta_{1}}=\frac{b}{2} \sum_{n=0}^{N} a_{n} \tau^{n}(1-\tau)^{1 / 2}(1+\tau)^{\beta_{1}}
$$


and by following the procedure described in Section 4 above with the collocation points given in (60). The tables also show the exact values of the stress intensity factors calculated from (69) and (70). It is seen that for $\mu_{2}<\mu_{1}$ excellent agreement is obtained by using only six terms in (72). However, for $\mu_{2}>\mu_{1}$ Table 2 shows that $\beta_{2}-\beta_{1}-1<0$ and, as indicated in the previous section, one would expect the convergence to be slow. This may indeed be seen from Tables 3 and 4 .

Referring to Section 4, for $\mu_{2}>\mu_{1}$ we now define the unknown function $w$ in (8) as follows:

$$
\begin{aligned}
w(t)= & g(t)(b-t)^{1 / 2} t^{\beta_{1}} \\
= & \frac{b}{2}\left[(1-\tau)^{1 / 2}(1+\tau)^{\beta_{1}} \sum_{n=0}^{N_{1}} a_{n} \tau^{n}+(1-\tau)^{1 / 2}(1+\tau)^{\beta_{2}} \sum_{n=0}^{N_{2}} b_{n} \tau^{n}\right] \\
& (0<t<b, \quad-1<\tau<1) .
\end{aligned}
$$

The collocation points are again selected as the roots of Chebyshev polynomials, namely

$$
\rho_{j}=\cos \left(\frac{2 j+1}{N_{1}+N_{2}+2} \frac{\pi}{2}\right) \quad\left(j=0,1, \ldots,\left(N_{1}+N_{2}+1\right)\right) .
$$

TABLE 3. Normalized stress intensity factors for a crack terminating at the interface (anti-plane shear) $(N+1=6$ terms are used in the expansion (72)).

\begin{tabular}{|l|rr|rc|}
\hline \multirow{2}{*}{$\frac{\mu_{2}}{\mu_{1}}$} & \multicolumn{2}{|c|}{$\bar{k}_{3}(0)$} & \multicolumn{2}{|c|}{$\bar{k}_{3}(b)$} \\
\cline { 2 - 5 } & & (Exact) & & (Exact) \\
\hline 0.04333 & 0.0656 & 0.0656 & 1.2558 & 1.2558 \\
0.1 & 0.1396 & 0.1396 & 1.1940 & 1.1940 \\
0.25 & 0.3101 & 0.3100 & 1.1144 & 1.1144 \\
0.5 & 0.5576 & 0.5575 & 1.0958 & 1.0958 \\
1. & 1.0000 & 1.0000 & 1.0000 & 1.0000 \\
2. & 1.8036 & 1.8134 & 0.9598 & 0.9598 \\
4. & 3.2694 & 3.3558 & 0.9334 & 0.9334 \\
10. & 7.0560 & 7.8231 & 0.9146 & 0.9146 \\
23.077 & 13.6113 & 17.3673 & 0.9067 & 0.9067 \\
40. & 20.3490 & 29.6029 & 0.9040 & 0.9040 \\
\hline
\end{tabular}

TABI.E 4. Stress intensity factor at the interface, $\frac{\mu_{2}}{\mu_{1}}=23.077$.

\begin{tabular}{cll}
$\frac{N+1}{6}$ & & $\frac{\bar{k}_{3}(0)}{13.61}$ \\
10 & & 14.49 \\
12 & 14.76 \\
16 & 15.14 \\
20 & 15.36 \\
25 & 15.59 \\
\hline Ref. [6] & 13.13 \\
Ref. [11] & 14.0 \\
\hline Exact & 17.37
\end{tabular}


For two values of $\mu_{2} / \mu_{1}$ the results obtained by using the expansion given in (73) are shown in Table 5. It is seen that without the second terms in (73) the convergence is very slow, whereas with the second term included very accurate results can be obtained even with a few terms in each series.

As a second example we consider the plane elasticity problem described by Fig. 1 and equations (12)-(17). In this case the characteristic equations are given by (42a, b). Examination of (42b) and the results given in [8] show that in all material combinations considered $\beta_{1}$ is real and $\beta_{2}-\beta_{1}>1$. No convergence difficulty is therefore expected. The stress intensity factors at the crack tips are defined by

$$
\begin{aligned}
& k_{1}(0)=\lim _{r \rightarrow 0} \sqrt{2} r^{1-\beta_{1}} \sigma_{2 \theta \theta}(r, 0), \\
& k_{1}(b)=\lim _{r \rightarrow b} \sqrt{2(r-b)} \sigma_{1 \theta \theta}(r, \pi) .
\end{aligned}
$$

By using (18), (20), (41), (28), and (36), from (75) and (76) we obtain

$$
\begin{gathered}
k_{1}(0)=\mu_{2} \frac{\beta_{1}}{\sin \pi \beta_{1}} g(0) \sqrt{2 b}\left[\left(\frac{3}{m+\kappa_{2}}-\frac{1}{1+m \kappa_{1}}\right)+2\left(1-\beta_{1}\right)\left(\frac{1}{1+m \kappa_{1}}-\frac{1}{m+\kappa_{2}}\right)\right], \\
k_{1}(b)=\frac{2 \mu_{1}}{1+\kappa_{1}} \frac{b^{\beta_{1}}}{\sqrt{2}} g(b) .
\end{gathered}
$$

\begin{tabular}{|c|c|c|c|}
\hline \multirow{5}{*}{$\frac{\mu_{2}}{\mu_{1}}=4$} & $N_{1}+1$ & $N_{2}+1$ & $\bar{k}_{3}(0)$ \\
\hline & 6 & 0 & 3.2694 \\
\hline & 10 & 0 & 3.3086 \\
\hline & 2 & 1 & 3.3149 \\
\hline & 3 & 1 & 3.3420 \\
\hline \multirow{5}{*}{$\begin{aligned}\left(\beta_{1}\right. & =0.70483 \\
\beta_{2} & =1.29517)\end{aligned}$} & 3 & 3 & 3.3550 \\
\hline & 5 & 2 & 3.3553 \\
\hline & 10 & 1 & 3.3553 \\
\hline & 10 & 2 & 3.3558 \\
\hline & \multicolumn{2}{|c|}{ EXACT } & 3.3558 \\
\hline \multirow{12}{*}{$\frac{\mu_{2}}{\mu_{1}}=23.077$} & $N_{1}+1$ & $N_{2}+1$ & $\bar{k}_{3}(0)$ \\
\hline & 6 & 0 & 13.61 \\
\hline & 10 & 0 & 14.49 \\
\hline & 25 & 0 & 15.59 \\
\hline & 1 & 1 & 14.89 \\
\hline & 2 & 1 & 16.62 \\
\hline & 2 & 2 & 17.11 \\
\hline & 5 & 1 & 17.26 \\
\hline & 3 & 3 & 17.35 \\
\hline & 4 & 4 & 17.35 \\
\hline & 5 & 10 & 17.36 \\
\hline & \multicolumn{2}{|c|}{ EXACT } & 17.37 \\
\hline
\end{tabular}

TABLE 5. Normalized stress intensity factors for a crack terminating at the interface (improved results). 
For a uniform crack, surface pressure $p(r)=p_{0}$ and for one material pair, the plane strain and plane stress results are given in Table 6 . The normalized stress intensity factors shown in the table are defined by

$$
\bar{k}_{1}(0)=\frac{k_{1}(0)}{p_{0}(b / 2)^{1-\beta_{1}}}, \quad \bar{k}_{1}(b)=\frac{k_{1}(b)}{p_{0} \sqrt{b / 2}} .
$$

The results shown in Table 6 were obtained by using ten terms in the series given in (72) and no convergence difficulties were encountered in the calculations.

In conclusion one may note that the technique would be readily applicable to more complicated problems involving, for example, finite dimensions, multiple cuts and general nonsymmetric loading conditions. In this case the coupled system of singular integral equations would have Fredholm as well as generalized Cauchy kernels and the fundamental functions may again be obtained by using the complex function technique described in this paper. Also, one should again emphasize the importance of examining the second (and subsequent) roots of the characteristic equations and, if necessary, taking them into consideration from the viewpoint of the convergence of the calculations and the accuracy of the results.

TABLE 6. Normalized stress intensity factors for a crack terminating at the interface (normal loading).

\begin{tabular}{l|l|c|c|}
\cline { 3 - 4 } \multicolumn{2}{c}{ Epoxy-Aluminum } & $\bar{k}_{1}(0)$ & $\bar{k}_{1}(b)$ \\
\hline \multirow{2}{*}{$\frac{\mu_{2}}{\mu_{1}}=23.077$} & $\begin{array}{l}\text { Plane strain } \\
\beta_{1}=0.6619\end{array}$ & 2.7997 & 0.8826 \\
\cline { 2 - 4 } & $\begin{array}{l}\text { Plane stress } \\
\beta_{1}=0.7110\end{array}$ & 4.2321 & 0.8787 \\
\hline \multirow{2}{*}{ Aluminum-Epoxy } & $\begin{array}{l}\text { Plane strain } \\
\beta_{1}=0.1752\end{array}$ & 0.0981 & 1.3421 \\
\cline { 2 - 4 }$\frac{\mu_{2}}{\mu_{1}}=0.0433$ & $\begin{array}{l}\text { Plane stress } \\
\beta_{1}=0.1758\end{array}$ & 0.0955 & 1.3398 \\
\hline
\end{tabular}

\section{REFERENCES}

[1] I. N. Sneddon, Mixed houndary value problems in potential theory, North Holland, Amsterdam, 1966

[2] N. I. Muskhelishvili, Singular integral equations, Noordhoff, Groningen. The Netherlands, 1953

[3] F. Erdogan, Mixed boundary value problems, Mechanics Today, S. Nemat-Nasser, ed. Vol. 4, 1-86 (1978)

[4] F. Erdogan and G. D. Gupta, On the numerical solution of singular integral equations, Quart. Appl. Máth. 29. $525-534$ (1972)

[5] A. C. Kaya and F. Erdogan, On the solution of integral equations with strongly singular kernels, Quart. Appl. Math. 45, 105-122 (1987)

[6] F. Erdogan and T. S. Cook, Antiplane shear crack terminating at and going through a bimaterial interface, Int. Journal of Fracture 10, 227-240 (1974)

[7] H. F. Bueckner, On a class of singular integral equations. J. Math. Analysis and Applications 14, 392-426 (1966)

[8] T. S. Cook and F. Erdogan, Stresses in bonded materials with a crack perpendicular to the interface. Int. J. Engng. Sci. 10, 667-697 (1972) 
[9] T. W. Chou, Dislocation pileups and elastic cracks at a bimaterial interface, Metallurgical Transactions 1, 1245-1248 (1970)

[10] P. S. Theocaris and N. I. Ioakimidis, Stress intensity factors at the tips of an antiplane shear crack terminating at a bimaterial interface, Int. Journal of Fracture 13, 549-552 (1977)

[11] J. L. Bassani and F. Erdogan, Stress intensity factors in bonded half planes containing inclined cracks and subjected to antiplane shear loading, Int. Journal of Fracture 15, 145-158 (1979) 